Check for updates

Cite this: RSC Adv., 2017, 7, 53025

Received 6th September 2017

Accepted 5th November 2017

DOI: 10.1039/c7ra09921e

rsc.li/rsc-advances

\section{Flexible grid-mesh electrodes fabricated by electroless copper plating on corona-treated PET substrates and coating with graphene for transparent film heaters $\dagger$}

\begin{abstract}
Bu-Jong Kim, Jong-Seol Park, Ria Yoo and Jin-Seok Park (D) *
In this study, we present solution-based processes for producing copper ( $\mathrm{Cu}$ ) meshes which can be utilized as transparent conductive electrodes (TCEs) for flexible film heaters. The surface modification of polyethylene terephthalate (PET) substrates was done via corona treatment at atmospheric pressure and room temperature. The $\mathrm{Cu}$ layer was deposited on the corona-treated PET substrate via electroless plating and then patterned via lithography to have mesh dimensions of a $200 \mu \mathrm{m}$ line-to-line spacing and a $6 \mu \mathrm{m}$ line width. Also, graphene was coated on the Cu mesh via electrophoretic deposition (EPD). The chemical and physical changes in the PET surfaces were characterized according to the corona treatment conditions. The measurements of contact angles and surface energies of the corona-treated PET substrates indicated that the PET surfaces changed from hydrophobic to hydrophilic after corona treatment, leading to the improvement in the adhesion between the PET substrates and the Cu meshes. The flexibility of the $\mathrm{Cu}$ meshes was inspected by performing bending and twisting tests and by directly measuring the adhesion strength between the $\mathrm{Cu}$ layers and the PET substrates through scratch tests. The effects of graphene coating on the characteristics of the $\mathrm{Cu}$ meshes were examined in terms of their surface morphologies, electrical sheet resistances, transmittances and reflectances in the visiblelight wavelength range, and color differences. Finally, the film heaters produced by employing the graphene-coated $\mathrm{Cu}$ meshes yielded a temperature rise over $85^{\circ} \mathrm{C}$ with a response time shorter than $20 \mathrm{~s}$.
\end{abstract}

\section{Introduction}

Transparent conductive electrodes (TCEs) have been utilized for a variety of applications, including touch screen panels, ${ }^{1,2}$ solar cells, ${ }^{3,4}$ film heaters, ${ }^{5,6}$ and displays. ${ }^{7,8}$ Up to now, thin films of indium-tin-oxide (ITO) as TCEs have been the most widely used. However, ITO is not compatible with the flexible versions of these devices due to its brittle nature as well as a high processing temperature. ${ }^{9}$ This has recently motivated research for finding alternative materials for ITO films, such as carbon nanotubes (CNTs), ${ }^{10,11}$ graphene, ${ }^{12,13}$ conductive polymers, ${ }^{14,15}$ metal nanowires, ${ }^{16,17}$ and metal meshes. ${ }^{18,19}$

Among the aforementioned materials, metal meshes have potential for commercialization due to their low electric sheet resistance and high visible transmittance. However, metal meshes inherently have a relatively high reflectance generated

Department of Electronic Systems Engineering, Hanyang University at ERICA Campus, 1271, Sa 3-dong, Sangnok-gu, Ansan, Gyeonggi-do 426-791, Republic of Korea. E-mail: jinsp@hanyang.ac.kr

$\dagger$ Electronic supplementary information (ESI) available: The photos of Cu films on PET substrates, the variations in the sheet resistances of $\mathrm{Cu}$ films, the wetting energy, the schematic of corona treatment system and corona energy density. See DOI: $10.1039 / \mathrm{c} 7 \mathrm{ra09921e}$ by the metals. Furthermore, several metals, such as gold (Au) and copper $(\mathrm{Cu})$, are generally tinged specific colors, leading to a visibility problem. In order to solve the poor visibility problems, metals need to be coated with a black material capable of absorbing light effectively. Graphene is known as an excellent absorber of visible light and has advantages in terms of chemical stability, thermal and electric conductivity, mechanical strength, and flexibility. ${ }^{20}$

Meanwhile, most of the currently-developed metal meshes have been fabricated by sputtering, which incurs high manufacturing costs due to vacuum and complicated processes. Recently, a variety of solution processes are adopted to produce metal meshes as they are simple and relatively inexpensive. ${ }^{21}$ However, the solution processes have common problems regarding the weak adhesion between the flexible substrates and the coating materials. ${ }^{22}$ Generally, plasma treatment has been widely used as the surface modification method to improve the adhesion between the flexible substrates and the coating layers formed on them. ${ }^{23}$ This method includes the chemical modification by which polar groups can be formed on the surface of substrate before film deposition, and also offers the physical change which can increase the area of surface via the formation of nano-scratches. The plasma treatment, 
however, forcibly demands several processing requirements such as low working pressures, long treatment times, and high process temperatures.

In this study, we present solution-based processes for fabricating $\mathrm{Cu}$ meshes on the polyethylene terephthalate (PET) substrates, which can be employed as the TCEs for flexible film heaters. Prior to the deposition of the $\mathrm{Cu}$ layer, the surface modification of the PET substrates was done via corona treatment at atmospheric pressure and room temperature. The $\mathrm{Cu}$ layers were deposited on the corona-treated PET substrates via electroless plating and then coated with graphene via electrophoretic deposition (EPD). The chemical and physical changes in the PET surfaces were characterized according to the corona treatment conditions such as the number of treatments, the treatment times, and the applied corona energy density. The flexibility of the $\mathrm{Cu}$ meshes was inspected by performing bending and twisting tests, and by directly measuring the adhesion strength between the Cu layers and the PET substrates through scratch tests. The effects of graphene coating on the characteristics of the $\mathrm{Cu}$ meshes were examined in terms of their surface morphologies, electrical sheet resistances, transmittances and reflectances in the visible-light wavelength range, and color differences. Finally, the thermal characteristics of film heaters produced in this study by employing the graphene-coated $\mathrm{Cu}$ meshes were measured using an infrared ray camera.

\section{Results and discussion}

\section{Surface modification of PET substrates via corona treatment}

The surface images of liquid drops that varied with the contact angles $\left(\theta_{c}\right)$ of the PET substrates are compared in Fig. 1(a)-(f), according to the numbers of corona-treatment. Two kinds of liquids such as water and diiodomethane were used in the $\theta_{\mathrm{c}}$ measurement.

The estimated $\theta_{\mathrm{c}}$ values are displayed Fig. 2(a). The $\theta_{\mathrm{c}}$ values based on water were seen to have monotonically decreased by increasing the number of corona-treatment. The opposite situation was discovered in the case of the $\theta_{\mathrm{c}}$ values measured with the diiodomethane-base liquid. From the $\theta_{\mathrm{c}}$ values, the surface energies of the PET substrates were also calculated using the

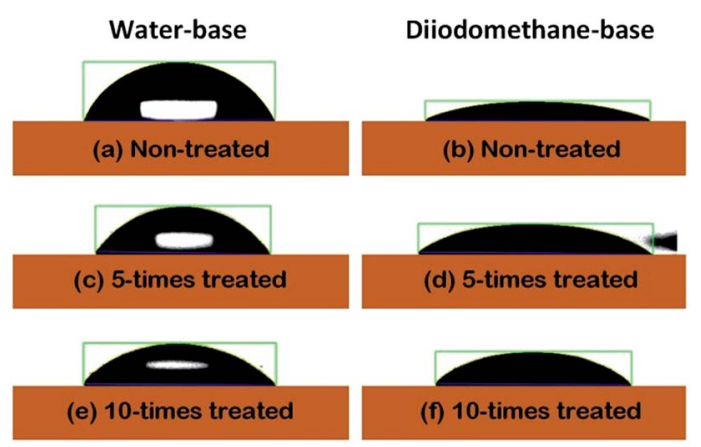

Fig. 1 The surface images of the liquid drops, such as water and diiodomethane, on the surfaces of the PET substrates as a function of the repetition numbers of the corona treatment.
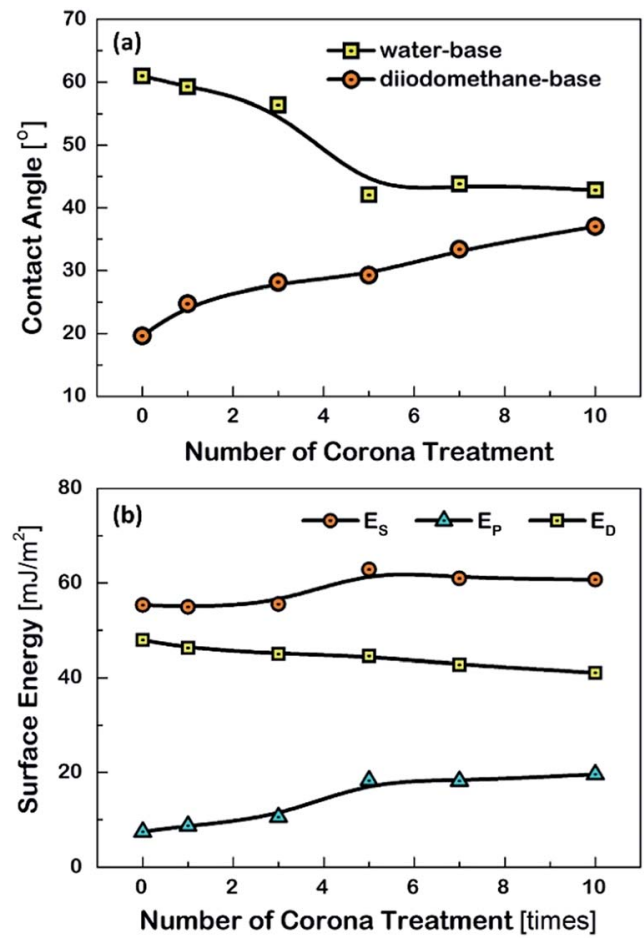

Fig. 2 (a) The estimated contact angles and (b) the surface energy of the PET substrates, as a function of the repetition numbers of the corona treatment.

formula in the Owens-Wendt's model. ${ }^{24}$ Fig. 2(b) shows the polar components (EP) calculated from the water-based $\theta_{\mathrm{c}}$ values, the dispersive components (ED) calculated from the diiodomethane-based $\theta_{\mathrm{c}}$ values, and the total surface energy (ES) obtained from the sum of EP and ED. The ES values were observed to have increased as the numbers of the corona treatment increased. This indicated that the chemical characteristic of the PET's surface was increasingly changing to a hydrophilic nature by increasing the numbers of coronatreatment. This was because molecular bonds on the surface of the PET substrate are broken during the corona treatment and the broken bonds are then recombined with free radicals in the corona environment to form polar groups on the PET'S surface. ${ }^{25}$ The related chemical analysis on this issue was reported in our previous study. ${ }^{26}$

It was also observed that the $\mathrm{Cu}$ films, when they were deposited on the non-treated PET or the once corona-treated PET substrate via electroless plating, were partially peeled off from the PET substrates as shown in Fig. S1(a) and (b). $\dagger$ On the other hand, the $\mathrm{Cu}$ films, when they were deposited on the corona-treated PET substrates more than 5 times, were well plated on the PET substrates without being peeled off, as shown in Fig. S1(c) and (d). $\dagger$ This was because the adhesive force between the PET substrates and the $\mathrm{Cu}$ films was increased by increasing the numbers of corona treatment, as had already been discussed with the surface energy.

In addition, the uniformities of the $\mathrm{Cu}$ films, which were deposited via electroless plating using the water-based solution, were examined by measuring the electric sheet resistances at 
the different measurement positions, as shown in the inset of Fig. S2(a). $\dagger$ The uniformity is described as the deviation of the sheet resistance measured at each position with respect to the average sheet resistance value (i.e., $\Delta R / R_{\text {ave }} \%$ ).

The results indicated that the uniformity was better for the $\mathrm{Cu}$ film deposited on the 10-times corona-treated PET substrate than that deposited on the 3-times corona-treated PET substrate. This may be related to the wettability of the PET substrate. $^{27}$ The result of Fig. S2(a) $\dagger$ indicated that the wettability of the PET substrate was improved by increasing the numbers of the corona treatment. The wetting energy of the PET substrate was also calculated from the water-based $\theta_{\mathrm{c}}$ value, as displayed in Fig. S2(b). $\dagger$ Increasing the numbers of corona treatment led to the increase in the wetting energy, indicating that the adhesion strength increased..$^{28}$

\section{Characteristics of $\mathrm{Cu}$ meshes fabricated on corona-treated PETs}

Fig. 3 shows the characteristics of the $\mathrm{Cu}$ meshes fabricated on the corona-treated PET substrates, such as the transmittance $\left(T_{550}\right)$ and reflectance $\left(R_{550}\right)$ measured in the wavelength of $550 \mathrm{~nm}$, and the sheet resistance $\left(R_{\mathrm{sh}}\right)$, as a function of the repetition numbers of the corona treatment. By increasing the treatment numbers from 3 times to 10 times, the $T_{550}$ slightly decreased from $91.5 \%$ to $90.4 \%$ and the $R_{550}$ increased from $3.2 \%$ to $4.8 \%$, and the $R_{\text {sh }}$ decreased from $21.2 \Omega \square^{-1}$ to 16.2 $\Omega \square^{-1}$. This may result from the difference in the areas

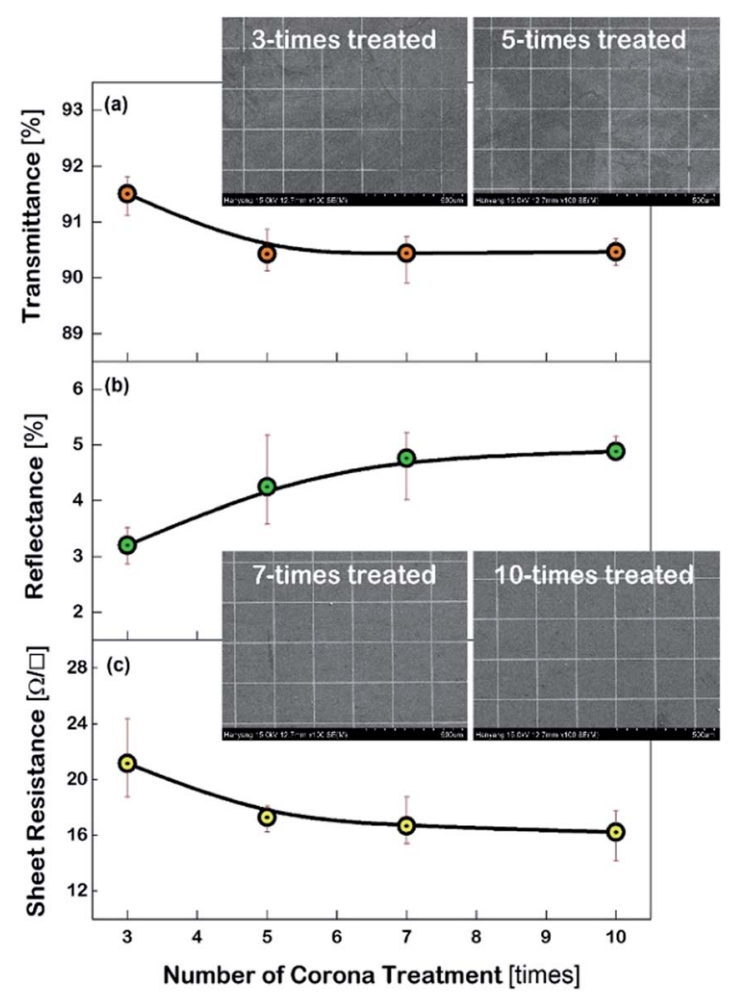

Fig. 3 The FESEM photos of the $\mathrm{Cu}$ meshes fabricated on the PET substrates as a function of the repetition number of the corona treatment of the PET substrates, and their characteristics, such as (a) transmittance, (b) reflectance, and (c) sheet resistance. where the $\mathrm{Cu}$ meshes were formed. As can be seen from the FESEM images of the $\mathrm{Cu}$ meshes shown in Fig. 3, for the $\mathrm{Cu}$ mesh deposited on the 3-times corona-treated PET substrate, the mesh patterns were partially broken or missing due to the relatively weak adhesion. For the $\mathrm{Cu}$ meshes deposited on the 7-times or 10-times corona-treated PET substrates, on the other hand, unimpaired mesh patterns without broken or missing patterns were observed.

The effect of corona-treatment on the flexibility of the $\mathrm{Cu}$ meshes deposited on PET substrates was also examined. The flexibility was quantitatively measured by recording the changes in the $R_{\mathrm{sh}}$ of the $\mathrm{Cu}$ meshes due to the application of repetitive bending and twisting. As can be seen from the photos shown in the insets of Fig. 4(a) and (b), the bending test was performed with the bending radius of $5 \mathrm{~mm}$ and the twisting test was carried out with the twisting angle of $30^{\circ}$.

As the numbers of bending and twisting increased, the $R_{\mathrm{sh}}$ values of the $\mathrm{Cu}$ meshes gradually increased. It should be noted, however, that the rise of the $R_{\mathrm{sh}}$ due to the bending or twist test was observed to have been substantially mitigated by increasing the numbers of corona treatment. For instance, the changes in the sheet resistance after 30000 times of bending with respect
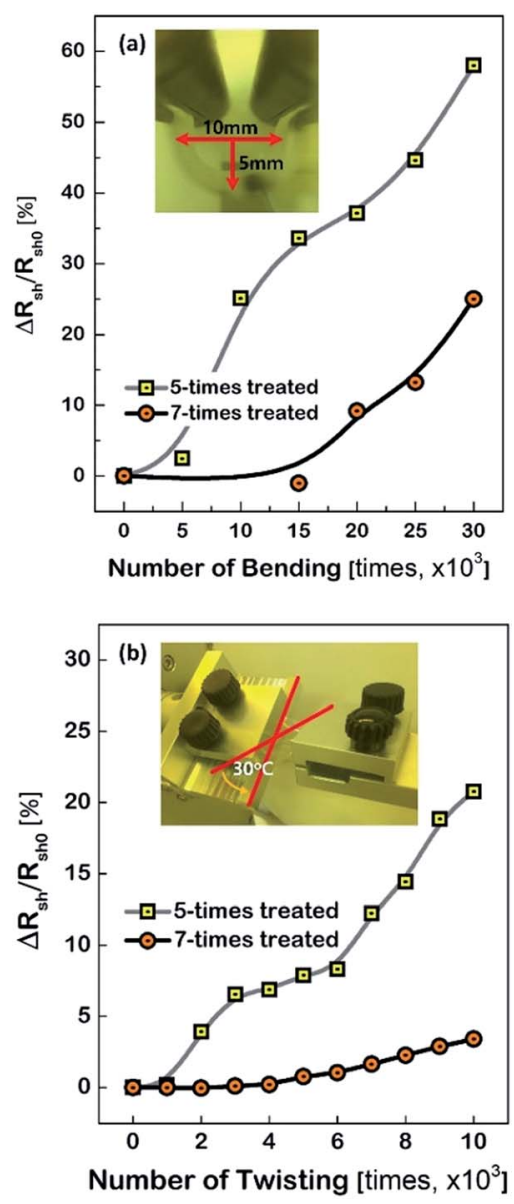

Fig. 4 The changes in the sheet resistances of the Cu meshes fabricated on the corona-treated (5-times and 7-times) PET substrates due to the flexibility tests, such as (a) bending test and (b) twisting test. 
to the initial sheet resistance (i.e., $\Delta R_{\mathrm{sh}} / R_{\mathrm{sh} 0}$ ) were measured to be approximately $58.0 \%$ for the $\mathrm{Cu}$ mesh fabricated on the 5 times corona-treated PET substrate and $25.0 \%$ for the $\mathrm{Cu}$ mesh fabricated on the 7-times corona-treated PET substrate. Also, the $\Delta R_{\mathrm{sh}} / R_{\mathrm{sho}}$ values after 10000 times of twisting were measured to be approximately $20.8 \%$ for the case of the 5 -times corona-treated PET substrate and only $3.4 \%$ for the case of the 7-times corona-treated PET substrate.

Generally, the electrode layers can be delaminated from the substrate due to the compressive force during the bending and twist test. Accordingly, the result that the $\mathrm{Cu}$ meshes fabricated on the more corona-treated PET substrates yielded the smaller increment of the $R_{\mathrm{sh}}$ after the flexibility tests was attributed to the strengthened adhesion between the $\mathrm{Cu}$ mesh and the PET substrate. To examine how the physical change of the PET substrate due to corona treatment affected the flexibility of the $\mathrm{Cu}$ mesh, the change in the surface roughness of the PET substrate was measured. The root-mean-square surface roughness $\left(\sigma_{\text {rms }}\right)$ values of the PET substrates were measured by increasing the numbers of corona treatment and the result is shown in Fig. 5(a), along with the AFM images of the nontreated and 10-times treated PET substrates. The non-treated PET substrate showed a relatively smooth surface with approximately $1.6 \mathrm{~nm}$ of $\sigma_{\text {rms }}$. In contrast, for the 10-times coronatreated PET substrate, the $\sigma_{\text {rms }}$ was significantly increased to about $6.6 \mathrm{~nm}$. This resulted from the generation of nanoscratches during corona treatment. Also, this indicated that
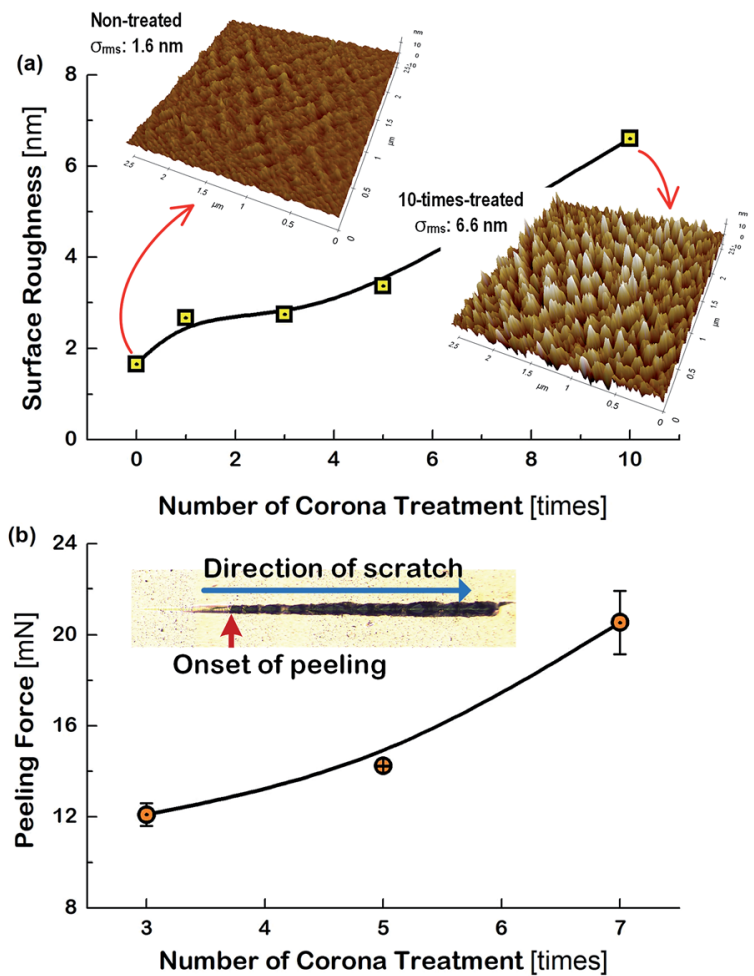

Fig. 5 (a) The surface roughness of PET substrates and (b) the peeling force of the Cu meshes fabricated on the PET substrates, as a function of the corona treatment times. The peeling force was defined as the applied load which caused the onset of peeling of Cu films. the rough surface of the PET substrate resulted in the increase of surface energy and also served as the anchor leading to higher adhesive strength between the $\mathrm{Cu}$ mesh and the PET substrate.

In addition, a scratch test was performed for the $\mathrm{Cu}$ sheets deposited on the corona-treated PET substrates by increasing the applied force to the specimens from $0.3 \mathrm{mN}$ to $50 \mathrm{mN}$. The results for the specimens that used the 3-times, 5-times, and 7times corona-treated PET substrates are displayed in Fig. 5(b).

The picture in the inset of Fig. 5(b) indicates the onset of peeling (i.e., peeling force) of the $\mathrm{Cu}$ sheet and the scratching direction. The peeling force increased from $12.1 \mathrm{mN}$ for the 3-times treated specimen to $20.5 \mathrm{mN}$ for the 7-times treated specimen, indicating the increase of the adhesive force between the PET substrate and the $\mathrm{Cu}$ sheet. This may be the mechanical evidence responsible for the observations of Fig. 4 that the flexibility of the $\mathrm{Cu}$ mesh could be improved by corona treatment of the PET substrate.

\section{Effects of graphene coating on $\mathrm{Cu}$ mesh}

Fig. 6 shows the FESEM photo of the $\mathrm{Cu}$ mesh coated with grapheme via electrophoretic deposition (EPD), along with the enlarged photo of the surface morphology of the graphene coating layer. It is clearly seen that graphene was selectively coated only on the surfaces where the $\mathrm{Cu}$ mesh patterns had been formed. The results of Fig. 7 show the changes in the $R_{\mathrm{sh}}$, $T_{550}, R_{550}$, and color difference $\left(C_{a b}^{*}\right)$ of the $\mathrm{Cu}$ meshes as a function of the EPD time used for the coating of graphene. Here, all of the graphene-coated $\mathrm{Cu}$ meshes were fabricated using the 7-times corona-treated PET substrates.

The $R_{\mathrm{sh}}$ was hardly affected regardless of graphene coating as shown in Fig. 7(a). The $T_{550}$ was slightly decreased by increasing the EPD time, but it still maintained higher than $85 \%$, as shown in Fig. $7(\mathrm{~b})$. Meanwhile, the $R_{550}$ was significantly reduced by graphene coating as shown in Fig. $7(\mathrm{c})$. For instance, the $R_{550}$ was about $4.7 \%$ for the $\mathrm{Cu}$ mesh without graphene coating and decreased to about $1.9 \%$ for the $\mathrm{Cu}$ mesh coated with graphene via $11 \mathrm{~s}$ of EPD. This indicated that the graphene coating layer played the role of suppressing the visible light reflected from the $\mathrm{Cu}$ mesh.

Generally, the color property of a metal mesh is closely related to its reflectance spectrum, from which the chromatic parameters, such as redness $\left(a^{*}\right)$ and yellowness $\left(b^{*}\right)$, can be

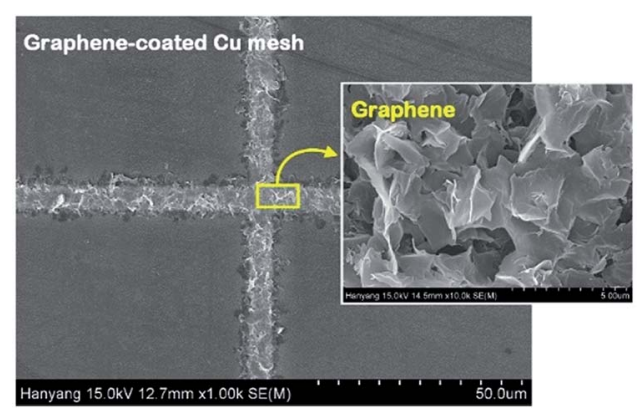

Fig. 6 The FESEM image of the graphene-coated Cu meshes, along with the surface morphology of the graphene layer. 


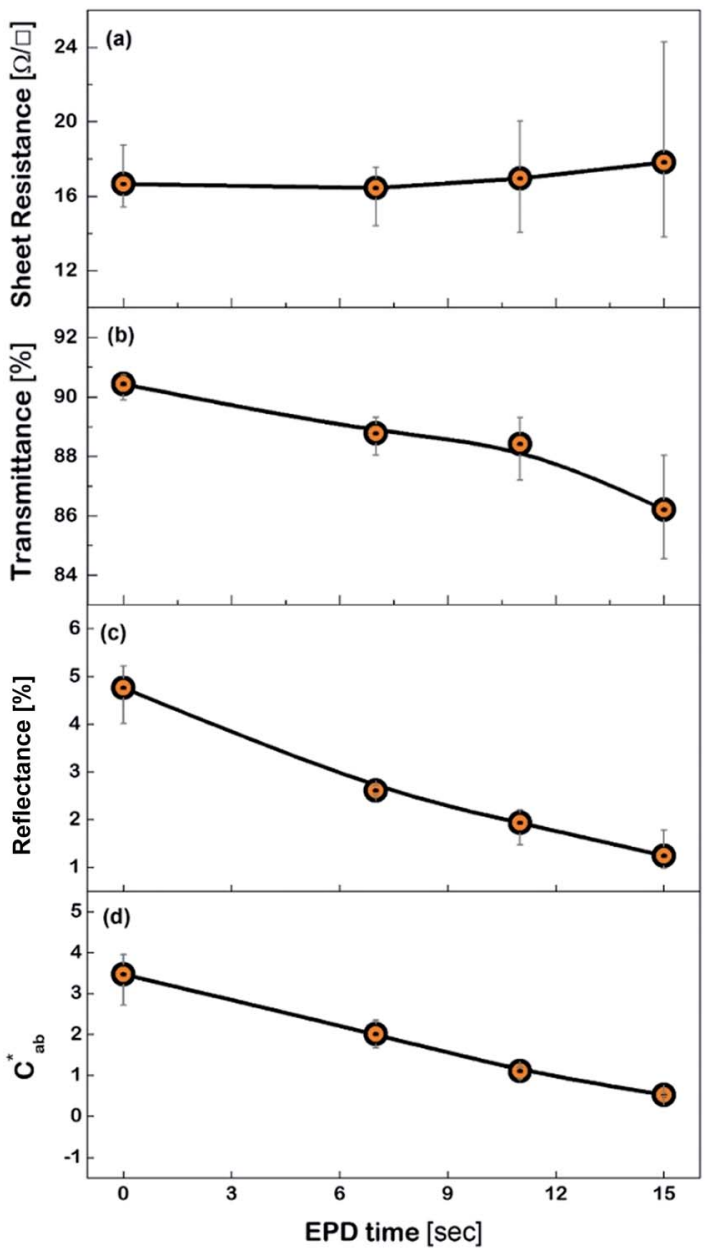

Fig. 7 The characteristics of the graphene-coated Cu meshes, such as (a) the sheet resistance, (b) the transmittance, (c) the reflectance, and (d) the color difference $\left(C_{a b}^{*}\right)$, as a function of the EPD time used for graphene coating.

determined. The value of parameter $a^{*}$ is the positive definiteness $(+)$ that comes close to the color red as it increases, and close to the color green as it increases with negative definiteness $(-)$. The value of parameter $b^{*}$ comes close to the color yellow as it increases with positive definiteness and appears to be blue as it increases with negative definiteness. ${ }^{29}$ Both the $a^{*}$ and $b^{*}$ components being closer to zero indicates that the metal mesh appears nearly colorless. Fig. 7(d) shows the color difference $\left(C_{a b}^{*}\right)$ which expresses the chromatic property of the metal mesh as the distance from the origin (i.e., $a^{*}=0$ and $\left.b^{*}=0\right)$ in the $a^{*}-b^{*}$ color coordinates..$^{30}$ As the EPD time increased, the $C_{a b}^{*}$ continuously decreased.

For instance, the $C_{a b}^{*}$ was about 3.47 for the $\mathrm{Cu}$ mesh without graphene coating and decreased to about 1.10 for the $\mathrm{Cu}$ mesh coated with graphene via $11 \mathrm{~s}$ of EPD. This implied that the visibility of the $\mathrm{Cu}$ mesh was improved by graphene coating due to the reduction of reflectance generated from the $\mathrm{Cu}$ mesh.

\section{Film heater using graphene-coated $\mathrm{Cu}$ mesh}

The graphene-coated $\mathrm{Cu}$ mesh electrodes fabricated on the corona-treated PET substrates were used to manufacture the flexible film heaters. Fig. 8(a) shows the temperature versus applied voltage relationship, along with the measurement schematic. The steady state temperature of graphene-coated $\mathrm{Cu}$ mesh with the $R_{\mathrm{sh}}$ value of about $15 \Omega \square^{-1}$ increased almost linearly with the applied voltage. This was based on Jouleheating: $P=V^{2} / R$, where $V$ is the applied voltage and $R$ the total resistance. The applied power density was calculated to be about $0.53 \mathrm{~W} \mathrm{~cm}^{-2}$ at the applied voltage of $5 \mathrm{~V}$.

Fig. 8(b) displays the temperature profile of the film heater when the input voltage of $5 \mathrm{~V}$ was applied, as a function of the operation time. The heating and cooling rates were calculated from the temperature derivatives, which were about $8.0^{\circ} \mathrm{C} \mathrm{s}^{-1}$ and $-7.6{ }^{\circ} \mathrm{C} \mathrm{s}^{-1}$ respectively.

The room temperature before turn-on was measured at the region A, which was about $29.9^{\circ} \mathrm{C}$. Also, the steady state
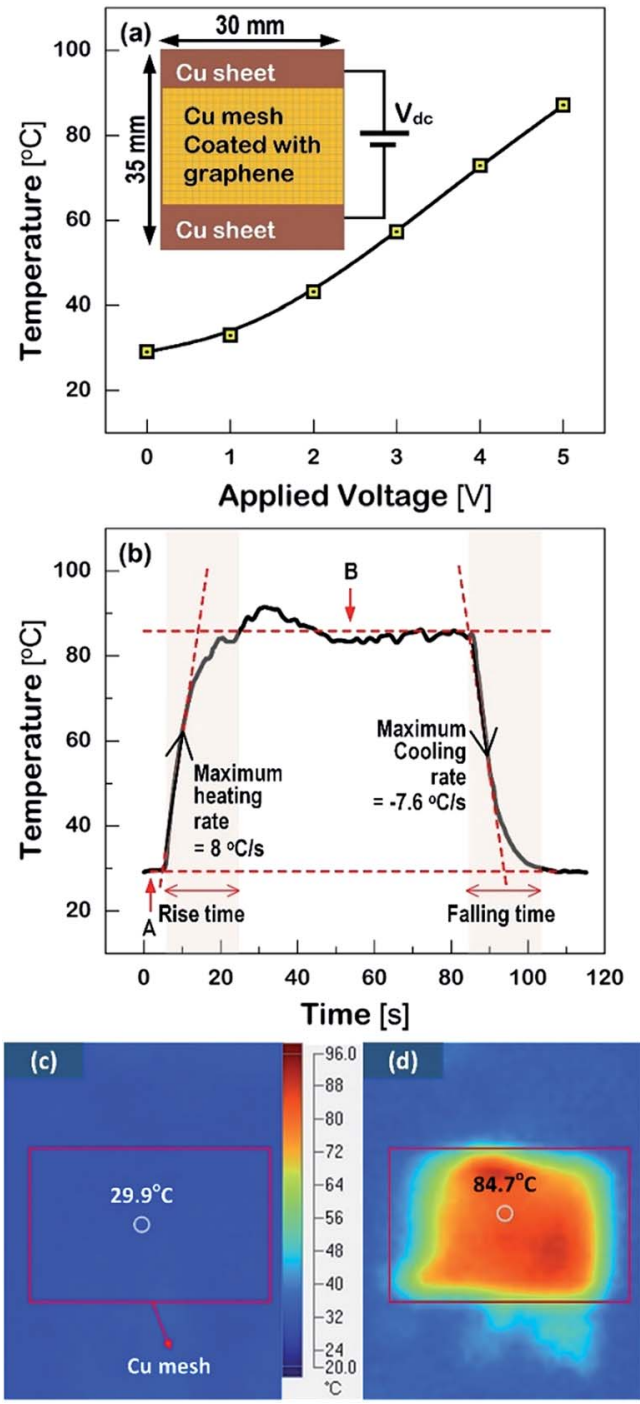

Fig. 8 The thermal characteristics of the fabricated graphene-coated Cu mesh electrodes: (a) the temperature-applied voltage relationship along with the measurement schematic. (b) The temperature profile of the electrode at the input voltage of $5 \mathrm{~V}$. [(c) and (d)] The thermal images at the input voltage of the A-region (before turn-on) and the Bregion (steady state), respectively. 
temperature was measured at the region $\mathrm{B}$, which was about $84.7^{\circ} \mathrm{C}$. Both the rise time after turn-on and the falling time after turn-off were shorter than $20 \mathrm{~s}$. This was due to the high heating and cooling rate of the $\mathrm{Cu}$ mesh electrode. The thermal images with the heating area of about $3.16 \mathrm{~cm}^{2}$ before and after operation are shown in Fig. 8(c) and (d) respectively. Accordingly, it is suggested that the graphene-coated $\mathrm{Cu}$ mesh electrodes fabricated in this study possess the improved visibility and high flexibility as well as the thermal characteristics, such as the heating capability of $85^{\circ} \mathrm{C}$ at $5 \mathrm{~V}$ and the response time of shorter than $20 \mathrm{~s}$, which would be desirable for flexible film heater application.

\section{Experimental}

\section{Method of corona-treatment}

Fig. S3† shows the schematic of the corona discharge system used in this study. The corona discharge was generated by applying $8 \mathrm{kV}$ voltage to a corona electrode with a width of $190 \mathrm{~mm}$. The PET substrate passed between two electrodes (i.e., the corona electrode and the grounded electrode) at a specified speed for surface treatment. The corona energy was controlled by varying the number of corona-treatment with the substrate feeding speed fixed at $0.027 \mathrm{~m} \mathrm{~s}^{-1}$, as also displayed in Fig. S3. $\dagger$

\section{Electroless plating of $\mathrm{Cu}$}

The electroless plating was progressed through several steps in order, such as cleaning, pre-dip, activation, reduction, and plating, by preparing the solutions (ROHAR-202, ROHAR470PD, ROHAR-470, ROHAR-570, and ROHAR-880, HANGARAM CHEMISTRY Co.) for each step. The organic contaminants were removed using a cleaner at $45{ }^{\circ} \mathrm{C}$ for $180 \mathrm{~s}$ before corona treatment. The corona-treated PET substrates were dipped into the pr-dip and activation solutions for the absorption of palladium (Pd) ions on the PET surfaces. Then, the Pd ions $\left(\mathrm{Pd}^{+2}\right)$ were reduced to $\mathrm{Pd}$ metal $\left(\mathrm{Pd}^{0}\right)$ used as the catalyst in the reduction process. Finally, the Pd served to reduce $\mathrm{Cu}$ ion $\left(\mathrm{Cu}^{2+}\right)$ on the PET surface in the plating process for $420 \mathrm{~s}$.

\section{Grid mesh pattering process}

$\mathrm{Cu}$ grid meshes were prepared via photolithography and chemical etching according to the following processes. A photoresist (PR) was deposited on the Cu film via spin coating for $1 \mathrm{~min}$ at $5000 \mathrm{rpm}$ to produce a $2.1-2.3 \mu \mathrm{m}$ thickness. Baking was performed for $180 \mathrm{~s}$ in a hot plate at $100^{\circ} \mathrm{C}$. The grid pattern was formed on the PR layer through exposing UV and developing. Then, the $\mathrm{Cu}$ film was dipped into the etching solution, which was prepared by mixing the acetic acid $\left(\mathrm{CH}_{3}\right.$ $\mathrm{COOH})$, hydrogen peroxide $\left(\mathrm{H}_{2} \mathrm{O}_{2}\right)$, and DI water at a $1: 1: 10$ volume ratio. Finally, the $\mathrm{Cu}$ layer was patterned to have a mesh dimension with a $200 \mu \mathrm{m}$ line-to-line spacing and a $6 \mu \mathrm{m}$ line width after stripping the residual PR.

\section{Graphene coating}

The graphene suspension (N-BaroTech, ER-4400) was prepared through the following purification and dispersion processes.
The graphene was placed in a mixture of $\mathrm{H}_{2} \mathrm{SO}_{4}(20 \mathrm{ml})$ and $\mathrm{HNO}_{3}(10 \mathrm{ml})$ at a $2: 1$ volume ratio and ultrasonicated for $30 \mathrm{~min}$ to eliminate impurities, such as metal catalysts and amorphous carbons. The graphene solution was filtered out using a vacuum filtering system and dispersed for 15 min using an ultrasonic generator after adding a dispersant of magnesium nitrate $\left(\mathrm{Mg}\left(\mathrm{NO}_{3}\right)_{2} \cdot 6 \mathrm{H}_{2} \mathrm{O}, 15 \mathrm{mg}\right)$ and isopropyl alcohol (IPA, $200 \mathrm{ml}$ ). The well-dispersed supernatant was extracted by operating a centrifugal separator at a $3000 \mathrm{rpm}$ speed for $10 \mathrm{~min}$. The graphene was coated on the $\mathrm{Cu}$ meshes deposited on the 7-times corona-treated PET substrates via EPD process, where a cathode ( $\mathrm{Cu}$ mesh) and an anode (ITO-coated glass) were immersed in the graphene suspension and an electric current of $2 \mathrm{~mA}$ was applied to the two electrodes with the electrode-spacing fixed at $5 \mathrm{~mm}$. The thickness of the graphene was controlled by varying the EPD time from $7 \mathrm{~s}$ to $15 \mathrm{~s}$.

\section{Characterization of PET substrates and $\mathrm{Cu}$ meshes}

The contact angles of the PET substrates were measured with both water as a polar liquid and diiodomethane as a nonpolar or dispersive liquid using dynamic contact angle (DCA, SEO, Phoenix 300) equipment. The atomic force microscopy (AFM, Park System, XE-100) was used to measure the change in the surface roughness of the PET substrates after corona treatment. The thickness of each layer in the electrode was measured using a stylus profilometer (BRUKER, Dektak XT). The field emission scanning electron microscopy (FESEM, Sigma, Carl Zeiss) was used to observe the surface morphology of PET substrates, Cu meshes, and graphene coating layers. The sheet resistance was measured using non-contact sheet resistance measurement equipment (NAPSON, EC-80P). The transmittance, reflectance, and chromatic parameters (e.g., redness, yellowness) were measured in the 300-700 $\mathrm{nm}$ wavelength range using a spectrum colorimeter (Konica Minolta, CM-5). Also, the flexibility of the mesh electrodes was inspected by performing a bending test (JUNIL Tech, JIBT-200) and custom-made twisting test (MIREA B \& S) equipment and also by measuring the adhesion strength between the $\mathrm{Cu}$ layers and the PET substrates using a scratch tester (Anton Paar, NST3). Finally, the thermal characteristics of film heaters employing the $\mathrm{Cu}$ meshes prepared in this study were measured using an infrared ray camera (Fluke, Tis10) by varying the input voltage from $1 \mathrm{~V}$ to $5 \mathrm{~V}$.

\section{Conclusions}

The graphene-coated $\mathrm{Cu}$ mesh electrodes were fabricated on the corona-treated PET substrates. The results of the contact angle and surface energy indicated that the surface condition of the PET substrates changed from hydrophobic to hydrophilic after corona treatment. It was also discovered that the surface roughness of the PET substrate was increased by increasing the number of corona treatments, which served as the anchor for strengthening the adhesive force between the PET substrate and the overlying $\mathrm{Cu}$ layer. Furthermore, the results regarding the changes in the sheet resistance due to the repetitive application of bending (up to 30000 times) and twisting (up to 10000 
times) confirmed that the flexibility of the $\mathrm{Cu}$ mesh electrodes was substantially improved by increasing the numbers of corona treatments for the PET substrates. Coating the Cu mesh with graphene significantly reduced the reflectance and yielded a color difference approaching zero (i.e., colorless), while it slightly decreased the transmittance. Finally, the flexible film heaters were manufactured by employing the $\mathrm{Cu}$-meshes, which were deposited on the corona-treated PET substrates and coated with graphene, and the film heaters revealed a heating capability of $85{ }^{\circ} \mathrm{C}$ or higher at the applied input voltage of $5 \mathrm{~V}$ and a response time of shorter than $20 \mathrm{~s}$.

\section{Conflicts of interest}

There are no conflicts to declare.

\section{Acknowledgements}

This research was supported by the BK21PLUS Program (No. 22A20130012328) and Basic Science Research Program through a National Research Foundation of Korea (NRF) (No. 2017R1D1A1B03028306).

\section{References}

1 C. H. Kim, W. J. Choi, S. K. Cho, D. Goshima, D. S. Ham, K. J. Kim, J. Jeong, J. H. Lee and S. J. Lee, Plasma Processes Polym., 2015, 12, 1322-1327.

2 Y. Kim, G. J. Jeon, M. K. Lee, S. H. Lee and S. H. K. Park, ECS Trans., 2016, 10, 247-251.

3 K. A. Bush, C. D. Bailie, Y. Chen, A. R. Bowring, W. Wang, W. Ma, T. Leijtens, F. Moghadam and M. D. McGehee, Adv. Mater., 2016, 28, 3937-3943.

4 C. Chen, L. Ling and F. Li, Nanoscale Res. Lett., 2017, 12, 1-7. 5 E. H. Ko, H. J. Kim, S. J. Lee, J. H. Lee and H. K. Kim, RSC Adv., 2016, 6, 46634-46642.

6 X. He, A. Liu, X. Hu, M. Song, F. Duan, Q. Lan, J. Xiao, J. Liu, M. Zhang, Y. Chen and Q. Zeng, Nanotechnology, 2016, 27, 475709.

7 C. H. Song, K. H. Ok, C. J. Lee, Y. Kim, M. G. Kwak, C. J. Han, N. Kim, B. K. Ju and J. W. Kim, Org. Electron., 2015, 17, 208215.

8 C. H. Moon, IEEE Trans. Plasma Sci., 2010, 38, 2445-2450.

9 M. Cann, M. J. Large, S. J. Henley, D. Milne, T. Sato, H. Chan, I. Jurewicz and A. B. Dalton, Mater. Today Commun., 2016, 7, 42-50.
10 K. J. Singh, T. J. Singh, D. Chettri and S. K. Sarkar, Optik, 2017, 135, 256-270.

11 B. J. Kim, S. H. Han and J. S. Park, Surf. Coat. Technol., 2015, 271, 22-26.

12 J. Huo, J. Wu, M. Zheng, Y. Tu and Z. Lan, Electrochim. Acta, 2016, 187, 210-217.

13 S. Ji, S. J. Kim, W. Song, S. Myung, J. Heo, J. Lim, K. S. An and S. S. Lee, $R S C A d v$., 2016, 6, 19372-19376.

14 X. Zhang, J. Wu, J. Wang, J. Zhang, Q. Yang, Y. Fu and Z. Xie, Sol. Energy Mater. Sol. Cells, 2016, 144, 143-149.

15 S. Xiao, C. Liu, L. Chen, L. Tan and Y. Chen, J. Mater. Chem. A, 2015, 3, 22316-22324.

16 A. T. Bellew, H. G. Manning, C. Gomes da Rocha, M. S. Ferreira and J. J. Boland, ACS Nano, 2015, 9, 1142211429.

17 K. Mallikarjuna, H. J. Hwang, W. H. Chung and H. S. Kim, RSC Adv., 2016, 6, 4770-4779.

18 D. Lordan, M. Burke, M. Manning, A. Martin, A. Amann, D. O'Connell, R. Murphy, C. Lyons and A. J. Quinn, ACS Appl. Mater. Interfaces, 2017, 9, 4932-4940.

19 W. Kim, S. Kim, I. Kang, M. S. Jung, S. J. Kim, J. K. Kim, S. M. Cho, J. H. Kim and J. H. Park, ChemSusChem, 2016, 9, 1042-1049.

20 T. Matsumoto, T. Koizumi, Y. Kawakami, K. Okamoto and M. Tomita, Opt. Express, 2013, 21, 30964-30974.

21 J. Song and H. Zeng, Angew. Chem., Int. Ed., 2015, 54, 97609774.

22 C. M. Gabardo, R. C. Adams-McGavin, B. C. Fung, E. J. Mahoney, Q. Fang and L. Soleymani, Sci. Rep., 2017, 7, 1-9.

23 A. Bouhamed, A. M. Kia, S. Naifar, V. Dzhagan, C. Müller, D. R. T. Zahn, S. Choura and O. Kanoun, Appl. Surf. Sci., 2017, 422, 420-429.

24 D. K. Owens and R. C. Wendt, J. Appl. Polym. Sci., 1969, 13, 1741-1747.

25 R. Shishoo, Plasma Technologies for Textiles, Elsevier Science, 2007.

26 S. H. Han, B. J. Kim and J. S. Park, Surf. Coat. Technol., 2015, 271, 100-105.

27 Z. Liu, J. Zhao, W. Xu, L. Qian, S. Nie and Z. Cui, ACS Appl. Mater. Interfaces, 2014, 6, 9997-10004.

28 M. Connor, J. E. Bidaux and J. A. E. Månson, J. Mater. Sci., 1997, 32, 5059-5067.

29 E. Becker, Color Res. Appl., 2002, 27, 456.

30 B. J. Kim, Y. J. Hwang and J. S. Park, Nanotechnology, 2017, 28, 165201. 